\title{
Art Students: Do They Really Draw What They Know about the Inner Body?
}

\author{
Rosário M. Dias ${ }^{1}$, Maria C. Pires ${ }^{1}$, Maria R. Carvalho ${ }^{1}$, Helcília D. Santos ${ }^{1}$, Ana Ferreira ${ }^{1}$, Samir Ahmad ${ }^{1}$, \\ Isabel Ritto $^{2} \&$ José G. Evangelista ${ }^{1}$ \\ ${ }^{1}$ Egas Moniz - Cooperativa de Ensino Superior, CRL, Monte de Caparica, Portugal \\ ${ }^{2}$ CIEBA - Faculdade de Belas-Artes, Universidade de Lisboa, Lisboa, Portugal \\ Correspondence: Maria do Rosário Dias, Egas Moniz, Cooperativa de Ensino Superior, CRL, Monte de Caparica, \\ Portugal.
}

Received: March 21, 2021 Accepted: April 23, 2021 Online Published: April 28, 2021

doi:10.5539/jedp.v11n1p60 URL: http://doi.org/10.5539/jedp.v11n1p60

\begin{abstract}
The aim of the present study was to examine seven case studies and ascertain whether the imagos internalized by students of Fine Arts, a Young Person and an Elderly Person can be reworked, after the students have been submitted to a course in Anatomy. In the present study, we have combined two methods - gathering written responses and drawings - and examined what students know about the organs they drew and used a content analysis grid to evaluate the mental representation of the interior of the body of both profiles (Young Person and Elderly Person), before and after academic training (Anatomy classes). The preliminary data collected provided a prima facie scenario for the existence of at least one sequencial comulative progression in the development of the art students drawings. However further research is needed to establish the extent to which this finding might apply beyond the tasks assigned in the present protocol.
\end{abstract}

Keywords: anatomy, fine art students, drawings, mental representation, education

\section{Introduction}

Theoretical and conceptual questions have been raised in the scientific literature about the concepts of body schema, body image and the mental representation of the interior of the body. Scientific literature strongly refers to the scarcity of published studies on the mental representation of subjects regarding the anatomy of the interior of the body (Reiss \& Tunnicliffe, 2001; Stein \& McNair, 2002). Reiss and Tunnicliffe (2001) denounce study limitations in the sense that language will inevitably be a mediator between the researcher and those who are researched and suggests different ways of gathering information about subjects' understanding of this kind of the interior of the body. However, despite the richness and variety of the methods used by researchers, most of them rely on either talking or writing methodologies (White \& Gunstone, 1992; Tunniclifle \& Reiss, 1999a, 1999b). While these approaches have their own specific advantages and disadvantages, the present study intended to use an approach which relied "less on words" (Reiss \& Tunnicliffe, 2001; Stein \& McNair, 2002) and more on drawings, hence resorting to Fine Arts students as case study subjects.

Previous studies developed by the Egas Moniz Multidisciplinary Research Center in Health Psychology (Dias, Duque, Neves, Soares, Cardoso, \& Carrão, 2006; Dias \& Neves, 2016; Dias, Grillo Evangelista, Naben, Ritto \& Ferreira, 2019) have also used drawings in the Health Education field. One of these studies (Dias, Grillo Evangelista, Naben, Ritto, \& Ferreira, 2019) intended to discern how Fine Art's students mentally represent the inner morphology of the human body of a Young Person and of an Elderly person. The results suggest anatomical differences in the pictorial representation of the Young Person and of the Elderly Person, namely in the accentuation of the curvatures of the vertebral column, retrusion of the lower jaw and muscle flaccidity (Dias, Grillo Evangelista, Naben, Ritto, \& Ferreira, 2019). Although the body schema was the same for all individuals, the body image was singularly depicted and linked to each individual's history, representing a synthesis of his idiosyncratic perceptions, experiences and particularities.

Another study has shown that pictorial representations of "Healthy Person" appear as an identification, for the gender of the participant is consistent with that of the human figure they portrayed in pictorial representations of a "Sick Person", the gender of the figure depicted is the opposite of that of the participant who sketched the drawing (Dias, Neves, Simões, Grillo Evangelista, \& Martins dos Santos, 2016). This showed how difficult it was for 
participants to identify themselves with the mental representation of a sick person.

The aim of the present study was to examine seven case studies and to ascertain whether the internalized body imagos of a Young Person and of an Elderly Person could be reworked by Fine Arts students after they have been submitted to Anatomy classes.

In a certain way we agree with Reiss et al. (2002) when they refer that a pupil in some cases normally learns that a number of organs are joined into a whole organ system. From a teaching point of view this means that rather than teaching pupils from the start about whole organ system and then going into more detail about constituent organs essentially a model of disassembly. As far as we can say we can do go bether to begin to teach in primary scholl the individual organs and then help children to learn that these are assembled into functional systems - this could be a model of assembly.

\section{Method}

With this article, we intend to present some partial results of a longitudinal and exploratory study, formatted in seven case studies ( 3 men and 4 women) in which it is intended to ascertain whether, the images internalized by the students who attended a Faculty of Fine Arts, of a Young Person and an Elderly Person could be re-elaborated, after the students had been submitted to a model of teaching the syllabus of an Anatomy course unit (test), in which the curricular objectives were specifically focused on syllabus contents of Artistic Anatomy.

Despite the parsimony that normally stigmatizes - case studies - as a research methodology in the area of social sciences, case studies are epistemologically in harmony with the experience of the subjects who are involved in them and, in this analytical record, for the subjects involved, the results are configured as a natural basis of generalization, representing, in the present study, a set of seven social units of a specific and temporally defined analysis (Vilelas, 2009: 141), in what constitutes according to the design of the study, as cases of holistic format (Dezin \& Lincoln, 2005; Stake, 2001; Fachin, 2001; Yin, 2002). It should also be noted that case studies fit into a qualitative approach and, despite the criticisms of some authors who frame them at the level of a reducing research methodology, considering them without objectivity, representativeness and rigor, in the sense of constituting themselves as a credible method of investigation, we emphasize only that, this research methodology, in fact, does not seem to provide a consistent basis in terms of scientific generalizations of the results obtained (Miles \& Huberman, 1994; Fachin, 2001; Yin, 2002).

There are several ways for gathering information about student's knowledge. Interviews or written tests with open-ended questions may effectively elicit students in-depth thinking, but they are difficult to quantify and sometimes subjective. In contrast, drawings have been considered as a simple research instrument that enables easy comparisons at an international level. We investigated relationships between the level of understanding shown by university students' written responses focused on the function of bodily organs (Dias \& Neves, 2016; Dias, Neves, Simões, Grillo Evangelista, \& Martins dos Santos, 2016; Dias, Grillo Evangelista, Naben, Ritto, \& Ferreira, 2019; Reiss \& Tunnicliffe, 2001).

\subsection{Identify Subsections}

In the present study, combining two methods - gathering written responses (Dias, Grillo Evangelista, Naben, Ritto \& Ferreira, 2019; Leach, Driver, Scott, \& Wood-Robinson, 1995) and drawings (Dias, Neves, Simões, Grillo Evangelista, \& Martins dos Santos, 2016; Reiss \& Tunnicliffe, 2001), we examined what students know about the organs they drew. The relation between respondent's knowledge gathering from the written responses and its drawing done by University Art Students is the central focus of this study (Tunnicliffe \& Reiss, 1999).

\subsection{Participant (Subject) Characteristics}

This study was conducted in Portugal, at a Higher Education Institution located in Lisbon. All subjects were Fine Arts' students aged between 18-23 years (three male subjects and four female subjects).

\subsection{Sampling Procedures}

In the present study, the participants were instructed to draw the interior of the human body at discretion, in profile view. For this purpose, they were asked to complete an investigation protocol which was divided into two sections, Section A and B. Section A called upon the elaboration of two drawings, one representing the interior of the body of a Young Person and the other representing the interior of the body of an Elderly Person. In this section participants were also instructed to characterize the human figures drawn according to age and gender. Drawings were captioned by the research team as soon as they were collected where doubt concerning drawings' peculiar representations was at stake. Moreover, in this section participants were asked to answer two open-ended questions: 1) "What is a young person?" and 2) "What is an elderly person?". 
Section B called upon the completion of a sociodemographic questionnaire and the answer to two additional questions: a) "Is this your first time attending the Anatomy Curriculum Unit?" and b) "Have you had any training in Human Anatomy?".

Subsequently, a pictorial content analysis grid was elaborated for the present study, in order to proceed with the analysis of 28 drawings collected, which combined demographic variables as well as drawings' content analysis variables. The grid encompassed 15 main categories: 1) Gender (of the subject and gender attributed to the drawing); 2) Investment in the Body Figure; 3) Age (of the subject and age attributed to the drawing); 4) Body Image; 5) Predominant Body System; 6) Skin; 7) Residual Body Systems; Anatomical Structure: 8) Head; 9) Neck; 10) Spine; 11) Trunk; 12) Upper Limbs; 13) Lower Limbs; 14) Predominance of Osteoarticular Pathology and 15) Joints. In addition to these categories, 67 analytical subcategories were developed to improve the content analysis of the collected drawings.

\subsection{Research Design}

Data was collected in two separate moments: i) on the first day of students attending the course unit of Human Anatomy (Phase I); and ii) in the end of the semester (Phase II). All participants gave their consent to participate in the study.

Each participant received a research protocol where they were invited to draw with graphite pencil the internal morphology of the human body of a Young person and of an Elderly person. Participants had 30 minutes to complete the task. Once both drawings were completed, participants were invited to characterize the drawings they had made according to age and gender, as well as to caption the components of the interior of the human body that were drawn. The drawings were then analysed with a content analysis grid created to evaluate the mental representation of the interior of the body of the Young Person and of the Elderly Person. Other qualitative data (i.e. open responses) were analysed through the content analysis of the written narrative. The quantitative data were later analysed with the software SPSS (Statistical Package for the Social Sciences), version 19.0 for Windows.

\section{Results and Discussion}

According to the results obtained from the content analysis carried out on the 14 drawings that constitute the 7 selected case studies, requested gave origin to two different profiles, the profile of the Young Person and the profile of the Elderly Person, distinguished by particular and antagonistic traits attributed to each pictorial representation.

\subsection{Profile of a Young Person}

The drawings of the Young Person collected in Phase I (before the academic training) were mostly representative of the male gender category (57.1\%), with an average age of 19 years, while in Phase II (after academic training) they were mostly representative of the female gender category (71.4\%) with an average age of 21years (Figures $1 \mathrm{~A}$ and $1 \mathrm{~B})$.
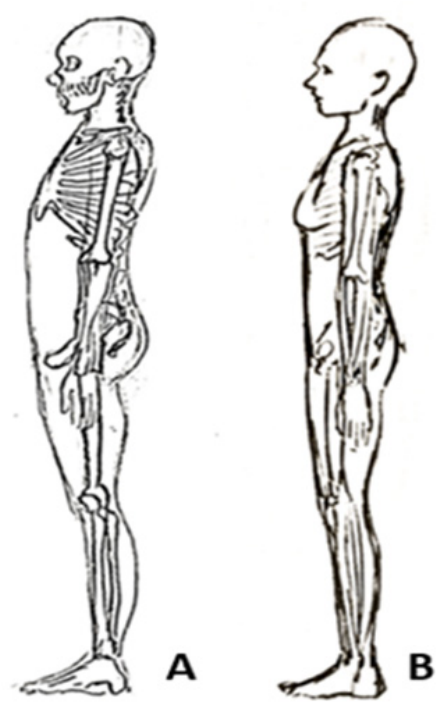

Figure 1. Examples of portraits drawn by subjects regarding the profile of a Young Person, before and after Human Anatomy classes. Portraits were first depited as of the male gender (A) and later on of the female gender (B) 
In the Investment in the Body Figure category in Phase I, drawings were mostly scored as Not Invested (57.1\%) (Figure 2), in stark contrast with Phase II drawings, which were mostly scored as Invested (85.7\%) (Figure 3).

In the Body Image category, drawings generally fell under the Normal subcategory (neither Fat nor Thin). In the Skin category, the human figures were outlined with Normal Skin $(71.4 \%)$ and with no signs of aging, (subcategories Flaccid or Wrinkled Skin), as illustrated in Figures 2E and 3F.

In the Predominant Body System category, the human figures represented mainly the Osteoarticular System subcategory (85.7\%), both on Phase I and Phase II (Figures 2 and 3). In these drawings it was noticable the absence of the Residual Body Systems category in the Phase I as well as the Gastro Intestinal System subcategory (14.2\%), in Phase II.

The Profile of the Young Person was represented mainly, at the level of - Anatomical Structure - with the frequency of $92.9 \%$ in the Neck category; the Head category is assumed, with dimensions proportional to the body, with the presence of the peripherical subcategories Maxilla (85.7\%), Mandible (78.6\%) and Teeth (50\%), with the absence of the Brain subcategory, as illustrated in Figures 2F and 3F. Aditionally, all Human Figures drawn by subjects, presented Normal or Slightly curved spine curvatures in the Spine category.

In the Trunk category only $42.8 \%$ of female human figures portrayed the Breast subcategory, both in Pase I and II. In all cases, breasts fell into the Normal and Not Saggy subcategories. In addition, the most common features represented pictorially in the Trunk category, fell into the subcategories Normal Abdomen (71.4\%), Spine (71.4\%), Ribs (71.4\%), Chest (85.7\%), Clavicle (78.6\%), and Sacrococcygeal Area (100\%) (Figure 3D).

In Phase I the subcategories Shoulder Blade (28.6\%) and Sternum (42.9\%) were sparsely represented, in stark contrast to the results obtained in Phase II, in which these subcategories were represented. This finding suggests an evolution in terms of the acquisition of body knowledge that was taught in the Anatomy curricular unit and an increased motivational investment made by subjects in the drawings (Figure 3E). The same happened in the Uper Limbs category, which accounted for some evolution in terms of the acquisition of body knowledge, as the subcategories Humerus (92.9\%), Cubitus (78.6\%), Radius (85.7\%) and Hand (78.6\%) were added to drawings in Phase II of the study (Figure 3G).

In the Lower Limbs category, the Femur (100\%), Patella (78.6\%), Tibia (100\%), Peronium (64.3\%) and Foot $(100 \%)$ subcategories were included in both Phase I and II (Figures $2 \mathrm{~F}$ and $3 \mathrm{~F}$ ). Most of the drawings (85.7\%) do not show any predominance of Osteoarticular Pathology category.

In the Joints category in Phase I, joints were designed in a rudimentary way (42.9\%) (Figure 2E) and only partially drawn with regular bone contour (57.1\%) (Figure 2C). Curiously, drawings obtained in Phase II showcased joints presented with regular bone contours $(100 \%)$ in all human figures (Figure 3).

The subcategories of the category Articulations, Temporomandibular Joint (TMJ, 35.8\%), Head / Neck (78.6\%), Shoulder (92.9\%), Elbow (92.9\%), Wrist (71.4\%), Fingers Interphalangeal (28.6\%), Coxofemoral (92.9\%), Knee $(92.9 \%)$ and Tibiotarsic (78.6\%), in general, with more incidence in Phase II, as illustrated in Figure 3F.
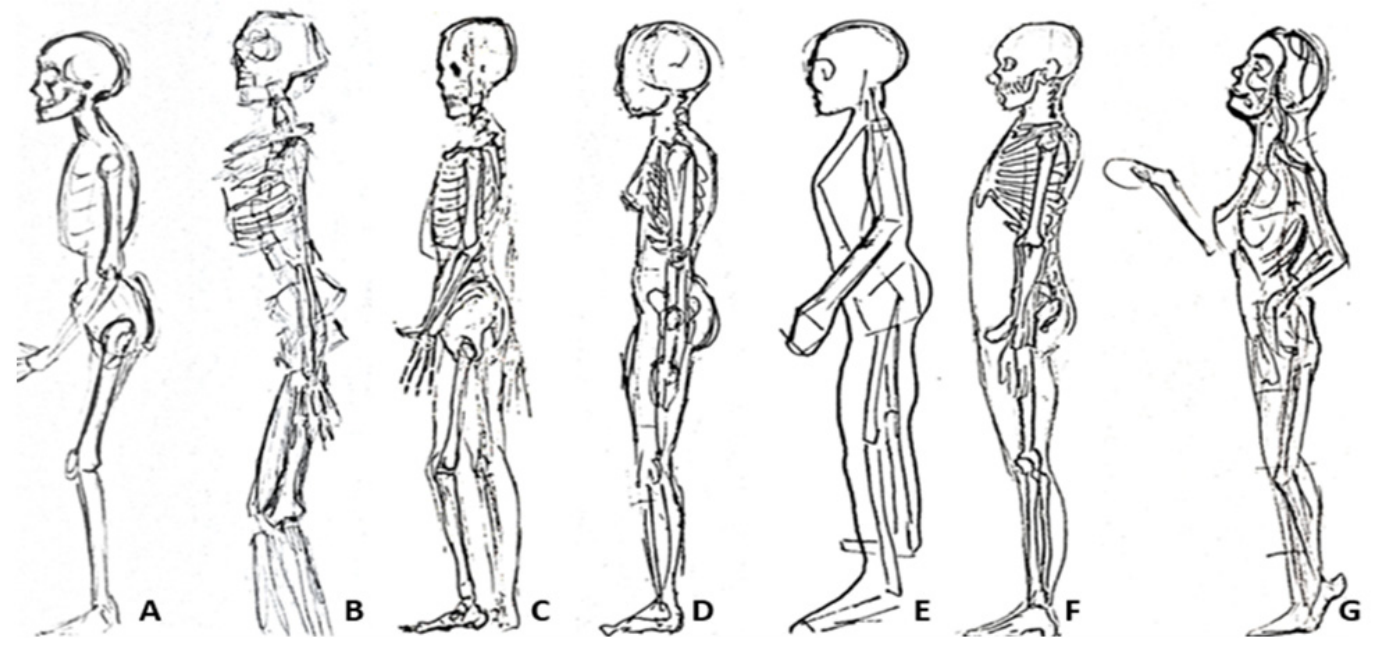

Figure 2. Examples of portraits drawn by subjects regarding the Profile of a Young Person in Phase I (before academic training) 

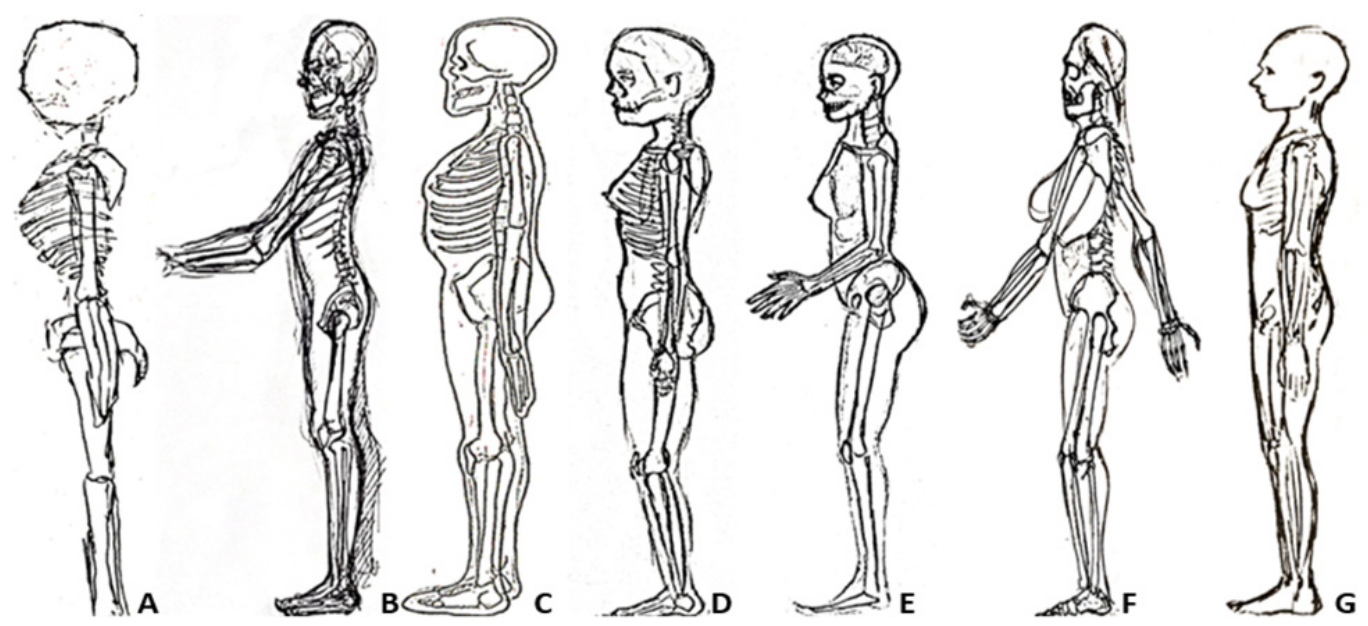

Figure 3. Examples of portraits drawn by subjects regarding the Profile of a Young Person in Phase II (after academic training)

Regarding the qualitative analysis of the open-ended questions (narrative), the average age atributed to the Profile of a Young Person was less than 45 years (35.8\%). Other attributes assigned by subjets to the Profile of a Young Person included, in order of prevalence 1) Vitality (including energetic, agile and with fast movements) $(57.1 \%) ; 2$ ) Healthy / Strong (35.7\%) and 3) Straight / Stable Posture (28.6\%), which seems to be in line with the results obtained in the content analysis of the drawings obtained.

\subsection{Profile of an Elderly Person}

The Profile of the Elderly Person in Phase I (Before Academic Training) was mostly represented as belonging to the male gender (57.1\%), with an average age of 68Y, while in Phase II (after academic training) it was mostly represented as belonging to the female gender category (71.4\%), with an average age of 63Y.

In the Investment in the Body Figure category the drawings were mostly Invested in both Phases $(71.4 \%)$. In the Body Image category, the drawings of the Human Figures denoted a body image that fell under the Normal subcategory $(42.9 \%)$ with a tendency towards the Slim subcategory in Phase I (28.6\%) (Figure 4E). In Pase II, drawings also denoted a body image that fell under the Normal subcategory $(71.4 \%)$ with a tendency towards the Fat subcategory (Figure 5E).

In the Skin category, 57.1\% of all human figures are outlined with Flaccid Skin in Phase I (Figure 4 (F)), as well as in Phase II (42.9\%). The subcategory Aged / Wrinkled skin was also frequently depicted in Phase II (28.6\%) (Figure 5G). The Predominant Body System category in both Phases I and II was the Osteoarticular System (92.9\%), also denoting some remnants of the Muscular System, the later only portrayed in Phase I (7.1\%) (Figure 4 D).

The Profile of the Elderly Person in both Phases I and II was represented mostly with the Anatomical Structure, Neck category $(92.9 \%)$. In the Head category, the vast majority of the heads drawn appeared with normal and proportional dimensions (71.4\%), with the presence of the Jaw (85.7\%), Mandible (85.7\%) and Teeth (50\%) subcategories and with the absence of the Brain subcategory (92.9\%), as illustrated in Figure 4G and Figure 5F.

In both Phases in the Trunk category, Saggy Breasts (42.9\%) were observed in the Breasts subcategory (57.1\%). The most common features in the Trunk category were in the Abdominal Prominence (71.4\%), Abdominal Recess (28.6\%), Spine (78.6\%) and Sacrococcygeal Area (85.9\%) subcategories. In Phase I, the majority of the figures drawn did not have Ribs (57.1\%), Rib Cage (57.1\%), Scapula (85.7\%), Clavicle (57.1\%) nor Sternum (85.7\%), whereas in Phase II the presence of the subcategories Ribs (71.4\%), Rib Cage (71.4\%); Scapula (71.4\%), Clavicle (57.1\%) and Sternum (85.7\%) were verified. Such suggests a slight advance in terms of the acquisition of body knowledge taught in the Anatomy curricular unit (Figures 4 B, C, D, F).

Regarding the Osteoarticular System, no significant differences were found between Phases I and II. In the Upper Limbs category, the Humerus (100\%), Cubitus (78.6\%), Radius (100\%) and Hand (92.9\%) were depicted, showing only a slight progress in terms of the knowledge acquired in the Anatomy curricular unit (Figure 3). In the Lower 
Limbs category, represented by the subcategories Femur (100\%), Patella (78.6\%), Tibia (100\%), Peronium $(64.3 \% \%)$ and Foot $(100 \%)$, most subjects $(85.7 \%)$ expressed pictorially a supremacy regarding the existence of Osteoarticular Pathology.

In the Joints category in Phase I, joints were drawn in a rudimentary way (57.1\%) (Figure 4 B, C, F) and with Irregular Bone Contours (28.6\%). However, in Phase II it was mostly represented by the Regular Bone Contours subcategory $(71.4 \%$ ) (Figure $5 \mathrm{G}$ ) and only $28.6 \%$ in a rudimentary way. Also represented in the Joints category and with no significant progress from Phase I to Phase II, were the TMJ (35.8\%), Head / Neck (85.7\%, Shoulder (85.7\%), Elbow (100\%), Wrist (85.7\%), Interphalangeal Hand (35.8\%), Coxofemoral (78.6\%), Knee (100\%) and Tibiotarsic (78.6\%) subcategories.
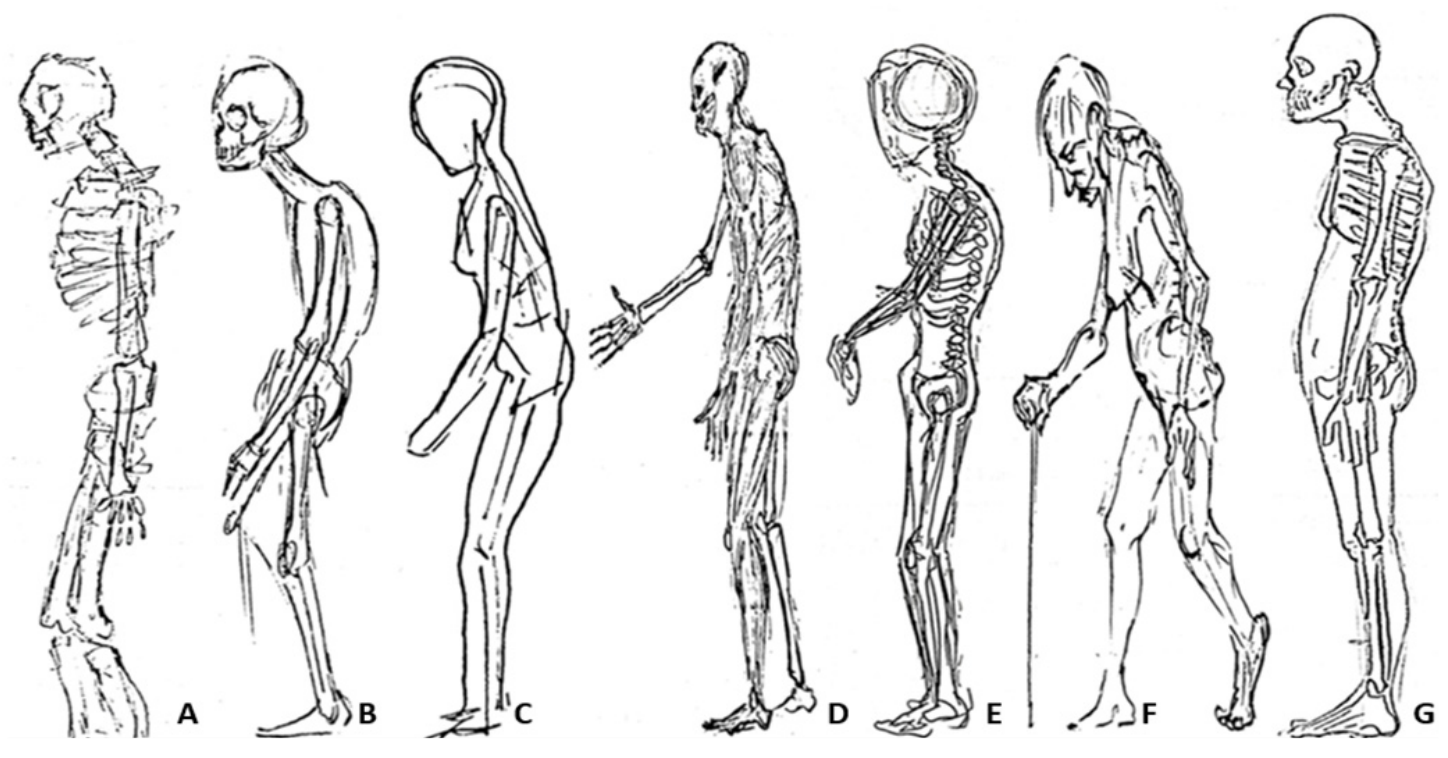

Figure 4. Examples of portraits drawn by subjects regarding the Profile of an Elderly Person in Phase I (before academic training)
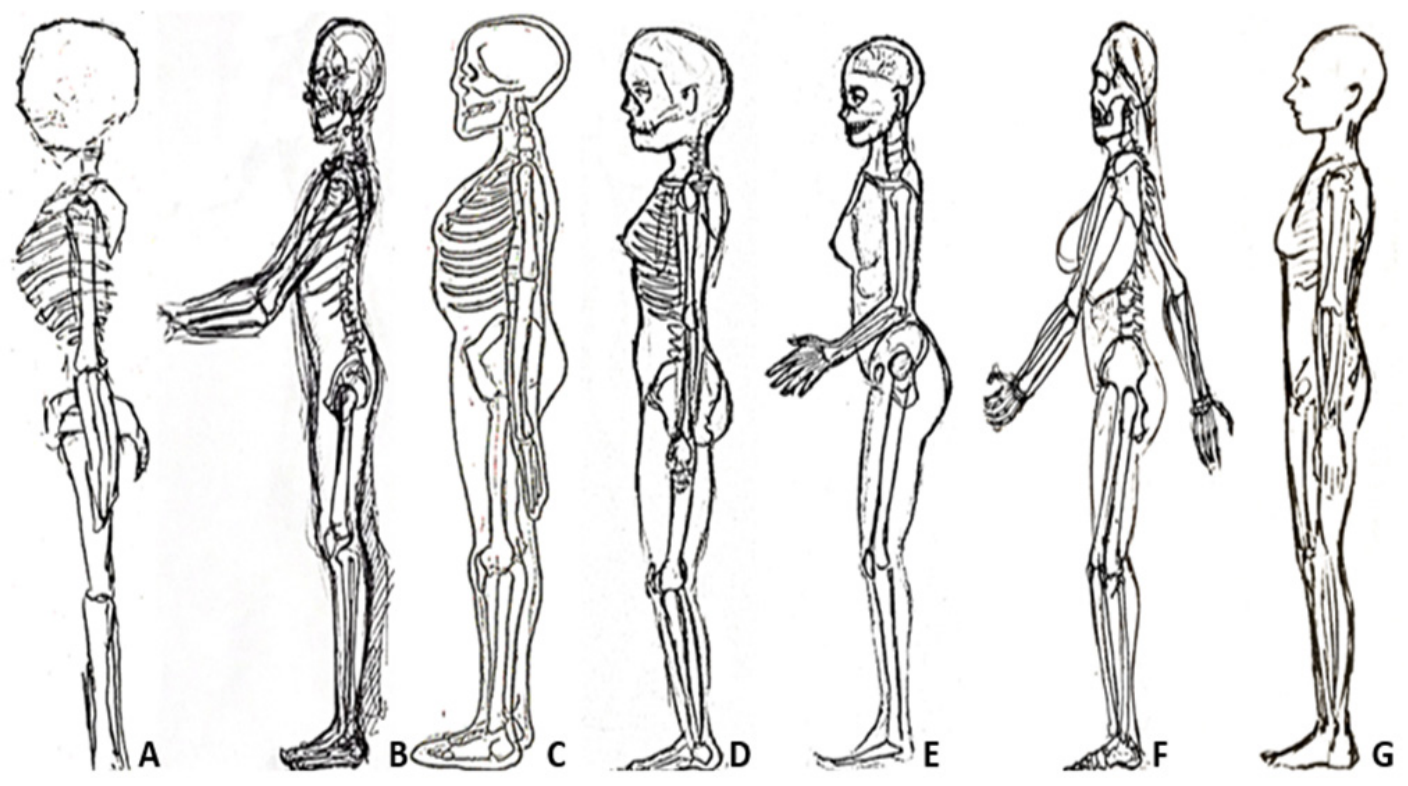

Figure 5. Examples of portraits drawn by subjects regarding the Profile of an Elderly Person in Phase II (after academic training) 
Regarding the qualitative answer analysis of the open-ended questions, the average age indicated to qualify the Profile of an Elderly Person is more than 60 years $(35,8 \%)$. Other features attributed by the participants of the study to the profile of an elderly person included, in order of prevalence: 1) Loss of Vitality (including loss of energy, tiredness and slow and rigid movements) (50\%); 2) Physical Weakness / Weak (28.6\%) and 3) Curved / Unstable Posture (21.5\%). Thus, according to the content analysis of the narrative of the answers, the attribute Physical Weakness / Weak seems to be more significant than the Osteoarticular Pathology category found in drawings. In parallel, the Curved / Unstable Posture attribute was in line with the pictorial representation of human figures in terms of the representation of a curved spine, in the Spine category.

\section{Conclusions}

Organs and body systems that are drawn more frequently are believed to be better understood than body systems that are not included in the drawings. In particular, a subsequent post-hoc test revealed that the Osteoarticular System acquired the highest relative score in comparison with other organ systems.

Instead, our data suggests that the students' concept about organ and body system location is independent from how these functions. In fact, organs drawn by students were generally well located, but the understanding of their function was usually lacking. According to the mean score of drawings, the majority of organs were drawn without relationships to one another (e.g. intestines were not connected with the stomach, lungs were not connected with the throat).

In the Profile of the Elderly Person, Human Figures are drawn with a hinting at tilting movements from the body which could be regarded as pseudo locomotion movements in the forward direction. In most Human Figures drawn as according to the Elderly Person Profile in Phases I and II, the presence of a lumbar lordosis and an accentuated thoracic kyphosis are generally identified. In addition, the representation of the gender attributed to the drawings in both profiles (young and elderly) was mainly masculine in Phase I and feminine in Phase II.

In fact, the subjects sampled for the present case study can be considered of high competence in terms of pictorial representation skills. Hence, the important prevalence of the Osteoarticular System in drawings seems to symbolically refer to this system as a structure / skeleton (a "hanger" of sorts) that supports the identity and representation of the body (as a puppet). Thus, the predominance of the Osteoarticular System as the "hanger" of the body is present both in the profile of the young and (even more so) of the elderly person. The tear of the osteocartilogeneous skeleton is portrayed with an accentuated curvature of the spine which is related to aging of the body. In addition, some drawings refer to this aging process via the presence of support tools (e.g. canes).

Despite the credibity of this study, the research methodology applied, does not seem to provide a consistent basis in terms of scientific generalizations of the results obtained.

\section{Acknowledgments}

The authors would like to dedicade this article to Professor José Martins dos Santos.

\section{References}

Denzin, N. K., \& Lincoln, Y. S. (2005). Handbook of Qualitative Research. Thousand Oaks: Sage.

Dias, M. R., \& Neves, A. C. (2016). Mente Sã em Corpo São: Representação mental do corpo saudável e doente na criança. Omnia: Revista Interdisciplinar de Ciências e Artes, 4, 55-64. https://doi.org/10400.26/13488

Dias, M. R., Duque, A. F., Neves, A. C., Soares, F. A., Cardoso, J. I., \& Carrão, L. M. (2006). Mente Sã em Corpo São. In J.L. Pais Ribeiro, I. Leal, and S. Jesus (Eds.). Actas do $6^{\circ}$ Congresso Nacional de Psicologia da Saúde (pp. 325-331). Lisboa: ISPA.

Dias, M. R., Grillo Evangelista, J., Naben, L., Ritto, I., \& Ferreira, A. (2019). X-Ray Vision: The Mental Geneaology of the Anatomy of the Interior of the Human Body. Journal of Biosciences and Medicine, 7 , 68-76. https://doi.org/10.4236/jbm.2019.78005

Dias, M. R., Neves, A. C., Simões, N. P., Grillo Evangelista, J., \& Martins dos Santos, J. (2016). X-Ray Vision: Mental representation of the human body's inner morphology. International Journal of Current Research, 8(6). https://doi.org/10400.26/14383

Fachin, O. (2001). Fundamentos de Metodologia. São Paulo: Saraiva.

Leach, J., Driver, R., Scott, P., \& Wood-Robinson, C. (1995). Children's ideas about ecology 1: Theoretical background design and methodology. International Journal of Science Education, 17(6), 721-732. https://doi.org/10.1080/0950069950170604 
Milles, M. B., \& Huberman, A. M. (1994). Qualitative Data Analysis. Thousand Oaks: Sage Publications.

Reiss, M. J., \& Tunnicliffe, S. D. (2001). Students' Understanding of Human Organs and Organ Systems. Research in Science Education, 31, 383-399. https://doi.org/10.1023/A:1013116228261

Reiss, M. J., Tunnicliffe, S. D., Andersen, A. M., Bartoszeck, A., Carvalho, G. S., Chen, S., .. \& Rooy, W. (2002). An international study of young people's drawings of what is inisde themselves. Journal of Biological Education, 36(2), 58-64. https://doi.org/10.1080/00219266.2002.9655802

Stake, R. E. (2001). The case study method in social inquiry. Educational Researcher, 7(2), 5-8. https://doi.org/10.3102/0013189X007002005

Stein, M., \& McNair, S. (2002). Science Drawings as a tool for analyzing conceptual understanding. In Association the Education of Teachers of Science Annual Meeting, Charlotte, North Carolina.

Tunnicliffe, S. D., \& Reiss, M. J. (1999). Student's understandings about animal skeletons. International Journal of Science Education, 21(11), 1187- 1200. https://doi.org/10.1080/095006999290147

Vilelas, J. (2009). Investigação: O processo de Construção do Conhecimento. Lisboa: Edições Sílabo.

White, R. T., \& Gunstone, R. F. (1992). Probing understanding. London: Falmer Press. https://doi.org/10.4324/9780203761342

Yin, R. K. (2002). Estudo de caso - Planejamento e Métodos. Porto Alegre: Bookman.

\section{Copyrights}

Copyright for this article is retained by the author(s), with first publication rights granted to the journal.

This is an open-access article distributed under the terms and conditions of the Creative Commons Attribution license (http://creativecommons.org/licenses/by/4.0/). 